ESAIM: PROCEEDINGS AND SURVEYS, October 2018, Vol. 64, p. 54-64

Laurence CARASSUS, Marion DARBAS, Ghislaine GAYRAUD, Olivier GOUBET, Stéphanie SALMON

\title{
SUPER-REPLICATION PRICE: IT CAN BE OK.
}

\author{
${\text { Laurence } \text { CARAssus }^{1} \text { And Tiziano VArgiolu }}^{2}$
}

\begin{abstract}
We consider a discrete time financial model where the support of the conditional law of the risky asset is bounded. For convex options we show that the super-replication problem reduces to the replication one in a Cox-Ross-Rubinstein model whose parameters are the law support boundaries. Thus the super-replication price can be of practical use if this support is not to large. We also make the link with the recent literature on multiple-priors models.

Résumé. Nous considérons un modèle financier à temps discret, où le support de la loi conditionnelle de l'actif risqué est borné. Nous montrons, pour une option dont la fonction de paiement est convexe, que le problème de surréplication se réduit à un problème de réplication parfaite dans un modèle Cox-Ross-Rubinstein, dont les paramètres sont les bornes du support de la loi. Ainsi, le prix de surréplication peut être utilisé en pratique, si ce support n'est pas trop grand. Nous faisons aussi le lien avec la littérature récente portant sur les modèles à croyances multiples.
\end{abstract}

\section{INTRODUCTION}

The problem of giving a price to a financial asset in the presence of uncertainty is central in the economic and financial theory. Uncertainty refers to Knightian uncertainty which distinguishes between the known unknown (risk) and unknown unknown (uncertainty), a concept first introduced in [16]. In different words, the agent cannot be certain about the choice of a given prior to model the outcome of a given situation. Thus, instead of specifying one "historical" or "real" probability measure, a set $\mathcal{Q}$ of probability measures is given.

In the context of financial markets and mathematical finance this is related to the development in a post2008 world of the so-called robust finance, where the notion of uncertainty is a way to approach the issue of model risk. The relevance of this idea is illustrated by the modeling of a financial asset's volatility uncertainty, which began to be studied more than twenty years ago (see for example [2]) and revisited more recently (see for instance [14]) : a set of probability measures $\mathcal{Q}$ that roughly speaking contains the laws of the asset prices for different volatility processes appears naturally. Interestingly this set is non-dominated, i.e. no probability measure determines the set of events that can happen or not. This increases significantly the mathematical difficulty as some of the classical tools of probability theory (conditional expectation or essential supremum) are ill-suited, since they are defined with respect to a given probability measure.

In this multiple-priors setting, the study of the classical problems of mathematical finance as no-arbitrage characterisation, pricing, maximisation of (worst case) expected utility have seen a strong reneweal of interest these last years: see [9] and the references therein for discrete time models.

${ }^{1}$ Léonard de Vinci Pôle Universitaire, Research Center, 92916 Paris La Défense and LMR, Université de Reims-Champagne Ardenne. Email: laurence.carassus@devinci.fr

${ }^{2}$ Department of Mathematics Tullio Levi Civita, University of Padova. Email: vargiolu@math.unipd.it

(C) EDP Sciences, SMAI 2018 
The present contribution, whose first two parts were written at the beginning of the 2000's, seems of particular interest in the light of the research of the latter five years. It shows that some non-dominated Knightian uncertainty can be very easily handled and concrete prices obtained without the (very) heavy theory needed in [9]. It also provides an interesting example for those non-dominated model theory whose underlying assumptions are not trivial to verify.

We start with a mono-prior, discrete time financial market consisting of one risky asset $S$ and one riskless bond normalized to one. It is well known that discrete time models are intrinsically incomplete and thus, as perfect replication is not always possible, the full hedging of risk goes through super-replication. The superreplication price is the minimal initial wealth needed to hedge without risk the contingent claim. It has been introduced in the binomial setup for transaction costs by [6]. In the present mono-prior context, under the no-arbitrage assumption the super-replication cost of an European contingent claim $H$ is the supremum of the expectation of $H$ computed under the risk-neutral probability measures. This so-called dual formulation of the super-replication price has been extensively studied and we refer to [15] and the references therein. Nevertheless it is well known that this dual formulation does not enable in general to effectively compute the super-replication price.

In [10], the authors consider a discrete time model and provide a closed formula in order to compute the super-replication cost of European and American style options and also the associated hedging strategies. In the case of European vanilla options, finding the super-replication price reduces to the computation of some concave envelope of the payoff function. For more general options, it involves recursive computations using again a kind of concave envelope. Note that the iterated concave envelopp has also been used by [5] in order to compute the super-replication price. The coefficients of the affine function which appears in the concave envelope give the hedging strategy. The formula comes from the dynamic programming principle and enlighten the crucial role played by the conditional distribution of the underlying risky asset. When this distribution admits a density with respect to the Lebesgue measure which is strictly positive over all the positive real line ${ }^{1}$, [10] provide effective computation for the super-replication price of European and American style exotic options (including Asian, Lookback or Barrier options) and show that those price are too high to be used in practice. For example the super-replication price of an European call option is equal to the initial price of the underlying risky asset and that the hedging strategy is just the "Buy and Hold" one.

Here, we focus on another class of models, namely those such that the support of the conditional distribution of the underlying (multiplicative) increments is bounded. This is of course the case for discrete models as tree models. It is also true in continuous models such that, conditionally to the information at time $t$, the distribution of $\frac{S_{t+1}}{S_{t}}$ is equivalent to the Lebesgue measure on $\left[d_{t+1}, u_{t+1}\right]$. This is in particular true if the regulator imposes some bounds on the maximal variation of the asset price in a given time interval (see for example the Limit Up/ Limit down plan of the US Securities and Exchange Commission). For options having convex payoff, we prove that the super-replication price is equal to the replication price in a Cox-Ross-Rubinstein model (see [12]), whose parameters are the maximum bounds of the law support. This means that the super-replication price is easy to compute and can be of practical use if the fluctuation interval is not large. This can be used in a reverse manner: observing the quotes of the asset prices together with the ones of the options, one could derive the bounds of the distribution. This will be addressed in [3], where similar results are obtained but without the no-arbitrage assumption and where numerical experiments are provided.

Finally, we make the link with the multiple-priors setup. We assume that, conditionally to the information at time $t$, the law of $\frac{S_{t+1}}{S_{t}}$ can be any law with support included in $\left[d_{t+1}, u_{t+1}\right]$. We prove that the cornerstone assumption of [9] is satisfied and that the multiple-priors No Arbitrage condition holds true. We finally show that the multiple-priors super-replication price is again equal to the replication price in a Cox-Ross-Rubinstein model whose parameters are $\left(d_{t}, u_{t}\right)_{\{t \in\{1, \ldots, T\}\}}$.

\footnotetext{
${ }^{1}$ This includes the following models observed at discrete time: Black-Scholes model, stochastic volatility models or models governed by a mix of Brownian motion and Poisson process.
} 
The paper is organized as follows. In Section 1, we describe the mono-prior financial model and recall the algorithm found in [10]. Then, in Section 2 we present and prove our results on the computation of the mono-prior super-replication price. Finally Section 3 contains the results in multiple-priors context.

\section{THE MONO-PRIOR FINANCIAL MODEL AND THE SUPER-REPLICATION ALGORITHM}

\subsection{Notations and definitions}

We consider a discrete time financial model with finite horizon $T$ and set $\mathcal{T}=\{0,1, \ldots, T\}$. The market consists of one riskless asset with price process normalized to one and one risky asset with real-valued price process $S=\left\{S_{t}, t \in \mathcal{T}\right\}$. The stochastic price process $\left(S_{t}\right)_{t \in \mathcal{T}}$ is defined on a complete probability space $(\Omega, \mathcal{F}, \mathbb{P})$ equipped with the filtration $\mathbb{F}=\left\{\mathcal{F}_{t}, t \in \mathcal{T}\right\}$, where the $\sigma$-field $\mathcal{F}_{t}$ is generated by the random variables $S_{0}, S_{1}, \cdots, S_{t}$. We make the usual assumptions that $\mathcal{F}_{0}$ is trivial and $\mathcal{F}_{T}=\mathcal{F}$. Here $\mathbb{P}$ represents the single prior of the agent. In this part we assume that there is no uncertainty on this prior.

Here the risk is only on the price process which is assumed to satisfy Assumption 1.1 below. Recall that the support of a generic probability measure $Q$ on $\mathcal{B}(\mathbb{R})$ (which will be denoted by $\operatorname{supp}(Q)$ ) is the smallest closed set $K$ such that $Q(K)=1$ and it is easy to see that for every $x \in \operatorname{supp}(Q)$ and for every $\varepsilon>0$, we have $Q(B(x, \varepsilon))>0$, where $B(x, \varepsilon)=\{y \in \mathbb{R},|y-x|<\varepsilon\}$ (see for example example [8, Ex. 12.9]).

Assumption 1.1. For all $t \in\{0,1, \ldots, T-1\}$, there exists real numbers $d_{t+1}$ and $u_{t+1}$ satisfying $d_{t+1}<1<$ $u_{t+1}$ and such that the convex hull of the support of the conditional law of $\frac{S_{t+1}}{S_{t}}$ with respect to $\mathcal{F}_{t}$ is equal to $\left[d_{t+1}, u_{t+1}\right]$.

Remark 1.2. - Two main types of price processes satisfy this assumption. The first one are processes such that, conditionally to $\mathcal{F}_{t}$, the distribution of $S_{t+1}$ is discrete and finite. Tree models are prototype of such models. The second family of models are the one such that, conditionally to $\mathcal{F}_{t}$, the distribution of $\frac{S_{t+1}}{S_{t}}$ is equivalent to the Lebesgue measure on $\left[d_{t+1}, u_{t+1}\right]$. Of course one can consider any combinations of both types.

- The no-arbitrage condition states that one cannot make money out of nothing (see the precise Definition 1.3). We will see in Lemma 1.4 that the condition $d_{t+1}<1<u_{t+1}$ is equivalent to the no-arbitrage assumption.

Next we define a trading portfolio by a $\mathbb{R}$-valued, $\mathbb{F}$-adapted process $\phi=\left\{\phi_{t}, t=0, \ldots, T-1\right\}$, where $\phi_{t}$ denotes the number of risky asset held at time $t$. The $\mathbb{R}$-valued $\mathbb{F}$-adapted process $C=\left\{C_{t}, t \in \mathcal{T}\right\}$ represents the cumulative consumption process. We assume that $C_{0}=0$ and that $C$ is non-decreasing. We also use the notation $\Delta S_{t}=S_{t}-S_{t-1}$ and $\Delta C_{t}=C_{t}-C_{t-1}$, for $t=1, \ldots, T$.

Given an initial wealth $x \in \mathbb{R}$, a trading portfolio $\phi$ and a cumulative consumption process $C$, the wealth process $X^{x, \phi, C}$ is governed by:

$$
\begin{aligned}
X_{0}^{x, \phi, C} & =x \\
X_{t}^{x, \phi, C} & =X_{t-1}^{x, \phi, C}+\phi_{t-1} \Delta S_{t}-\Delta C_{t}, \quad \text { for } t=1, \ldots, T .
\end{aligned}
$$

The possible condition $C=0$ means that the portfolio $\phi$ is self-financed and we will denote $X^{x, \phi}=X^{x, \phi, 0}$. The triple $(x, \phi, C)$ will be called a hedging strategy.

Following the presentation of [15], we recall basic definitions related to the super-replication prices. A European contingent claim will be represented by a $\mathcal{F}_{T}$-measurable random variable $H$. We denote by $\mathcal{A}_{H}^{e}$, the set of hedging strategies for $H$ such that $X_{T}^{x, \phi, C} \geq H \mathbb{P}$-a.s. Then, $(\hat{x}, \hat{\phi}, \hat{C}) \in \mathcal{A}_{H}^{e}$ is called minimal if for all $(x, \phi, C) \in \mathcal{A}_{H}^{e}, X_{t}^{x, \phi, C} \geq X_{t}^{\hat{x}, \hat{\phi}, \hat{C}} \mathbb{P}$-a.s. for all $t \in \mathcal{T}$. It is easy to see that $\hat{x}$ is then the so-called super-replication cost $p^{e}(H)$ of $H$, i.e. the minimal initial capital needed for hedging without risk $H$ :

$$
p^{e}(H)=\inf \left\{x \in \mathbb{R}: \exists(\phi, C) \text { s.t. }(x, \phi, C) \in \mathcal{A}_{H}^{e}\right\} .
$$

We now define the same notion for an American contingent claim $\left(H_{t}\right)_{t \in \mathcal{T}}$, where $H_{t}$ is a $\mathcal{F}_{t}$-measurable random variable for all $t \in \mathcal{T}$. Now $\mathcal{A}_{H}^{a}$ will be the set of American hedging strategies such that, for all $t \in \mathcal{T}$, 
$X_{t}^{x, \phi, C} \geq H_{t} \mathbb{P}$-a.s. Then $(\hat{x}, \hat{\phi}, \hat{C}) \in \mathcal{A}_{H}^{a}$ is minimal if for all $(x, \phi, C) \in \mathcal{A}_{H}^{a}, X_{t}^{x, \phi, C} \geq X_{t}^{\hat{x}, \hat{\phi}, \hat{C}} \mathbb{P}$-a.s., for all $t \in \mathcal{T}$. Again, $\hat{x}$ is the super-replication cost $p^{a}(H)$ of $H$, i.e

$$
p^{a}(H)=\inf \left\{x \in \mathbb{R}: \exists(\phi, C) \text { s.t. }(x, \phi, C) \in \mathcal{A}_{H}^{a}\right\} .
$$

Now, we introduce the set of equivalent martingale measures:

$$
\mathcal{P}=\left\{Q \sim \mathbb{P}: \frac{d Q}{d \mathbb{P}} \in L^{\infty}, \Delta S_{t} \in L^{1}(Q) \text { and } E^{Q}\left[\Delta S_{t} \mid \mathcal{F}_{t-1}\right]=0, \quad 1 \leq t \leq T \quad \mathbb{P} \text {-a.s. }\right\}
$$

Note that the Dalang-Morton-Willinger Theorem (see [13]) proves that the non-emptiness of $\mathcal{P}$ is equivalent to the economic meaningful assumption of no-arbitrage. This condition asserts that, starting from a zero wealth, it is not possible to reach a non-negative wealth which is strictly positive with strictly positive probability. The formal definition is given by:

Definition 1.3. There is no-arbitrage $N A(\mathbb{P})$ if $X_{T}^{0, \phi} \geq 0 \mathbb{P}$-a.s. implies that $X_{T}^{0, \phi}=0 \mathbb{P}$-a.s.

Lemma 1.4. If the convex hull of the support of the conditional law of $\frac{S_{t+1}}{S_{t}}$ with respect to $\mathcal{F}_{t}$ is equal to $\left[d_{t+1}, u_{t+1}\right]$ and $d_{t+1} \neq u_{t+1}$, the condition $d_{t+1}<1<u_{t+1}$ is equivalent to the no-arbitrage assumption. So under Assumption 1.1, NA( $\mathbb{P})$ condition holds true and $\mathcal{P} \neq \emptyset$.

Proof. Assume for example that $d_{t+1} \geq 1$. We buy the asset at time $t$ using a loan in the riskless asset, at $t+1$ we liquidate the positions and get $S_{t+1}-S_{t}$. As $\mathbb{P}\left(S_{t+1} \geq s_{t} d_{t+1} \mid S_{0}=s_{0}, \ldots, S_{t}=s_{t}\right)=1$, we get that $\mathbb{P}\left(S_{t+1}-s_{t} \geq 0 \mid S_{0}=s_{0}, \ldots, S_{t}=s_{t}\right)=1$. We want to prove that $\mathbb{P}\left(S_{t+1}-s_{t}>0 \mid S_{0}=s_{0}, \ldots, S_{t}=s_{t}\right)>0$. If this is not the case, $\mathbb{P}\left(S_{t+1} \leq s_{t} \mid S_{0}=s_{0}, \ldots, S_{t}=s_{t}\right)=1$ and by definition of the support $\left.\left.\left[d_{t+1}, u_{t+1}\right] \subset\right]-\infty, 1\right]$ which is either impossible or contradicts the assumption that $d_{t+1} \neq u_{t+1}$. For the reverse implication, assume that $\mathbb{P}\left(h\left(S_{t+1}-s_{t}\right) \geq 0 \mid S_{0}=s_{0}, \ldots, S_{t}=s_{t}\right)=1$ for some $h \in \mathbb{R}$. Assume first that $h>0$. Then $\mathbb{P}\left(S_{t+1} \geq s_{t} \mid S_{0}=s_{0}, \ldots, S_{t}=s_{t}\right)=1$ and thus $\left[d_{t+1}, u_{t+1}\right] \subset\left[1,+\infty\left[\right.\right.$ and $1 \leq d_{t+1}$, a contradiction. The case $h<0$ works similarly. Thus we must have $h=0$ and $\mathbb{P}\left(h\left(S_{t+1}-s_{t}\right)=0 \mid S_{0}=s_{0}, \ldots, S_{t}=s_{t}\right)=1$.

\subsection{Super-replication algorithm}

For the reader's convenience, we now recall the Carassus-Gobet-Temam (CGT) algorithm which allows to compute the super-replication price of derivative assets as presented in [10]. We start with the case of European option. For a measurable function $h$ from $\mathbb{R}^{T+1}$ into $\mathbb{R}$, we define the sequence of operators:

$$
\begin{aligned}
\Gamma_{T}^{e} h\left(s_{0}, \ldots, s_{T}\right) & =h\left(s_{0}, \ldots, s_{T}\right) \\
\Gamma_{t}^{e} h\left(s_{0}, \ldots, s_{t}\right) & =\operatorname{infess}_{\left\{(\alpha, \beta) \in I_{\Gamma_{t+1}^{e}}{ }_{t}\left(s_{0}, \ldots, s_{t}\right)\right\}}\left\{\alpha+\beta s_{t}\right\} \quad 0 \leq t \leq T-1
\end{aligned}
$$

where for a measurable function $v$ from $\mathbb{R}^{t+2}$ into $\mathbb{R}$ we define:

$$
I_{v}\left(s_{0}, \ldots, s_{t}\right)=\left\{(\alpha, \beta) \in \mathbb{R}^{2} \mid \mathbb{P}\left(\alpha+\beta S_{t+1} \geq v\left(s_{0}, \ldots, s_{t}, S_{t+1}\right) \mid S_{0}=s_{0}, \ldots, S_{t}=s_{t}\right)=1\right\}
$$

The essential infimum in (1.3) is related to the the law of the vector $\left(S_{0}, \ldots, S_{t}\right)$, which we indicate with $\mathbb{P}_{t}$. Then, the following theorem holds.

Theorem 1.5. Assume that $\mathcal{P} \neq \emptyset$. Let $H=h\left(S_{0}, \ldots, S_{T}\right)$ be a bounded from below European contingent claim, for some measurable function $h$ from $\mathbb{R}^{T+1}$ into $\mathbb{R}$. Assume that $\sup _{Q \in \mathcal{P}} E^{Q}[|H|]<\infty$. Then, there exists a minimal hedging strategy $(\hat{x}, \hat{\phi}, \hat{C}) \in \mathcal{A}_{H}^{e}$ and its value at time $t \leq T$ is

$$
X_{t}^{\hat{x}, \hat{\phi}, \hat{C}}=\Gamma_{t}^{e} h\left(S_{0}, \ldots, S_{t}\right) \mathbb{P}_{t}-\text { a.s. }
$$

In particular, $p^{e}(H)=\Gamma_{0}^{e} h\left(S_{0}\right)$. 
An analogous result holds in the American case. Consider a family of measurable functions $h=\left(h_{t}\right)_{t \in \mathcal{T}}$ such that for $t \in \mathcal{T}, h_{t}$ maps $\mathbb{R}^{t+1}$ into $\mathbb{R}$. We define a new sequence of operators $\Gamma^{a}$ as:

$$
\begin{aligned}
\Gamma_{T}^{a} h\left(s_{0}, \ldots, s_{T}\right) & =h_{T}\left(s_{0}, \ldots, s_{T}\right) \\
\Gamma_{t}^{a} h\left(s_{0}, \ldots, s_{t}\right) & =\max \left(\operatorname{infess}_{(\alpha, \beta) \in I_{\Gamma_{t+1}^{a}}^{a} h\left(s_{0}, \ldots, s_{t}\right)}\left\{\alpha+\beta s_{t}\right\}, h_{t}\left(s_{0}, \ldots, s_{t}\right)\right) \quad 0 \leq t \leq T-1,
\end{aligned}
$$

where the set $I_{v}$ has been defined in (1.4). Let $\mathcal{S}_{t, T}$ be the set of all stopping times w.r.t. the filtration $\mathbb{F}$ such that $t \leq \tau \leq T$.

Theorem 1.6. Assume that $\mathcal{P} \neq \emptyset$. Let $H=\left(H_{t}\right)_{t \in \mathcal{T}}$ be a bounded from below American contingent claim where $H_{t}=h_{t}\left(S_{0}, \ldots, S_{t}\right)$ and $h_{t}$ is a measurable function from $\mathbb{R}^{t+1}$ into $\mathbb{R}$ such that $\sup _{\tau \in \mathcal{S}_{0, T}, Q \in \mathcal{P}} E^{Q}\left[\left|H_{\tau}\right|\right]<\infty$. Then, there exists a minimal hedging strategy $(\hat{x}, \hat{\phi}, \hat{C}) \in \mathcal{A}_{H}^{a}$ and its value at time $t \leq T$ is

$$
X_{t}^{\hat{x}, \hat{\phi}, \hat{C}}=\Gamma_{t}^{a} h\left(S_{0}, \ldots, S_{t}\right) \mathbb{P}_{t}-a . s .
$$

In particular, $p^{a}(H)=\Gamma_{0}^{a} h\left(S_{0}\right)$.

For both European and American option, we also get that the optimal portfolio $\hat{\phi}$ is given step by step by the optimal $\beta$ from (1.3) and (1.6), see [10]

\section{EFFECTIVE COMPUTATION OF THE MONO-PRIOR SUPER-REPLICATION PRICE}

Now we present the main result of this section which asserts that when the support of the conditional law of the risky asset is bounded (see Assumption 1.1), the super-replication price of a convex option is equal to the replication price in a Cox-Ross-Rubinstein model whose parameters are the law support boundaries. First we prove the following lemma, which shows that it is only necessary to super-replicate a convex function at the boundary of the support.

Lemma 2.1. Let Assumption 1.1 holds true. For any convex function $v: \mathbb{R}^{t+2} \rightarrow \mathbb{R}$,

$$
I_{v}\left(s_{0}, \ldots, s_{t}\right)=\left\{(\alpha, \beta) \in \mathbb{R}^{2}, \alpha+\beta s_{t} x \geq v\left(s_{0}, \ldots, s_{t}, s_{t} x\right) \text { for } x \in\left\{d_{t+1}, u_{t+1}\right\}\right\} .
$$

Proof. Fix some $s_{0}, \ldots, s_{t} \in \mathbb{R}$. We prove the first inclusion $\supseteq$. Let $\omega \in \Omega$ be such that there exists $\lambda(\omega) \in[0,1]$ satisfying $S_{t+1}(\omega)=\lambda(\omega) s_{t} d_{t+1}+(1-\lambda(\omega)) s_{t} u_{t+1}$. Now, let $(\alpha, \beta) \in \mathbb{R}^{2}$ such that $\alpha+\beta s_{t} x \geq v\left(s_{0}, \ldots, s_{t}, s_{t} x\right)$ for $x \in\left\{d_{t+1}, u_{t+1}\right\}$. By convexity of $v$, we get that:

$$
\begin{aligned}
v\left(s_{0}, \ldots, s_{t}, S_{t+1}(\omega)\right) & \leq \lambda(\omega) v\left(s_{0}, \ldots, s_{t}, s_{t} d_{t+1}\right)+(1-\lambda(\omega)) v\left(s_{0}, \ldots, s_{t}, s_{t} u_{t+1}\right) \\
& \leq \alpha+\beta\left(\lambda(\omega) s_{t} d_{t+1}+(1-\lambda(\omega)) s_{t} u_{t+1}\right)=\alpha+\beta S_{t+1}(\omega) .
\end{aligned}
$$

Using Assumption 1.1, $\mathbb{P}\left(S_{t+1} \in\left[s_{t} d_{t+1}, s_{t} u_{t+1}\right] \mid S_{0}=s_{0}, \ldots, S_{t}=s_{t}\right)=1$ and we have that:

$$
\mathbb{P}\left(\alpha+\beta S_{t+1} \geq v\left(s_{0}, \ldots, s_{t}, S_{t+1}\right) \mid S_{0}=s_{0}, \ldots, S_{t}=s_{t}\right)=1 .
$$

For the reverse inclusion $\subseteq$, we argue by contradiction. Assume that for some $(\alpha, \beta) \in I_{v}\left(s_{0}, \ldots, s_{t}\right)$, we have that $\alpha+\beta s_{t} d_{t+1}<v\left(s_{0}, \ldots, s_{t}, s_{t} d_{t+1}\right)$. As $v$ is a convex function on $\mathbb{R}^{t+2}$, it is also continuous and there exists $\varepsilon$ such that $\alpha+\beta x<v\left(s_{0}, \ldots, s_{t}, x\right)$, for all $x \in B\left(s_{t} d_{t+1}, \varepsilon\right)$. From Assumption 1.1 $\mathbb{P}\left(\left|S_{t+1}-s_{t} d_{t+1}\right|<\varepsilon \mid S_{0}=s_{0}, \ldots, S_{t}=s_{t}\right)>0$ and we get that

$$
\mathbb{P}\left(\alpha+\beta S_{t+1}<v\left(s_{0}, \ldots, s_{t}, S_{t+1}\right) \mid S_{0}=s_{0}, \ldots, S_{t}=s_{t}\right)>0,
$$

a contradiction. The case $\alpha+\beta s_{t} u_{t+1}<v\left(s_{0}, \ldots, s_{t}, s_{t} u_{t+1}\right)$ works similarly. 
We are now able to compute explicitly the operators $\Gamma_{t}$ defined in (1.3) and (1.6). We begin with the European case.

Proposition 2.2. Let $H=h\left(S_{0}, \ldots, S_{T}\right)$ be a bounded from below European contingent claim, where $h$ : $\mathbb{R}^{T+1} \rightarrow \mathbb{R}$ is a convex function and $\sup _{Q \in \mathcal{P}} E^{Q}[|H|]<\infty$. Assume that Assumption 1.1 holds true. Then

$$
\begin{aligned}
\Gamma_{T}^{e} h\left(s_{0}, \ldots, s_{T}\right) & =h\left(s_{0}, \ldots, s_{T}\right) \\
\Gamma_{t}^{e} h\left(s_{0}, \ldots, s_{t}\right) & =\pi_{t+1} \Gamma_{t+1}^{e} h\left(s_{0}, \ldots, s_{t}, s_{t} u_{t+1}\right)+\left(1-\pi_{t+1}\right) \Gamma_{t+1}^{e} h\left(s_{0}, \ldots, s_{t}, s_{t} d_{t+1}\right)
\end{aligned}
$$

where $\pi_{t}:=\frac{1-d_{t}}{u_{t}-d_{t}}, t=1, \ldots, T$.

Proof. First we can use Theorem 1.5 since $\mathcal{P} \neq \emptyset$ (see Lemma 1.4). The proposition is proved by induction. Using (1.2) the result is true for $T$. Moreover $\Gamma_{T}^{e} h$ is convex by assumption. Now assume that for some $t \in\{1, \ldots, T-1\},(2.8)$ holds true and that $\Gamma_{t+1}^{e} h$ is convex. First, it is straightforward that $\Gamma_{t}^{e} h$ is a convex function from $\mathbb{R}^{t+1}$ into $\mathbb{R}$. From Lemma 2.1 , we get that

$$
\begin{aligned}
\Gamma_{t-1}^{e} h\left(s_{0}, \ldots, s_{t-1}\right) & =\operatorname{infess}_{\left\{(\alpha, \beta) \in \mathbb{R}^{2} \mid \alpha+\beta s_{t-1} x \geq \Gamma_{t}^{e} h\left(s_{0}, \ldots, s_{t-1}, s_{t-1} x\right), x \in\left\{d_{t}, u_{t}\right\}\right\}\left\{\alpha+\beta s_{t-1}\right\}} \\
& =\bar{\alpha}+\bar{\beta} s_{t-1},
\end{aligned}
$$

where $(\bar{\alpha}, \bar{\beta})$ are the unique $(\alpha, \beta)$ satisfying $\alpha+\beta s_{t-1} x=\Gamma_{t}^{e} h\left(s_{0}, \ldots, s_{t-1}, s_{t-1} x\right)$ for both $x=d_{t}$ and $x=u_{t}$, i.e.

$$
\left\{\begin{array}{l}
\bar{\alpha}=\frac{u_{t} \Gamma_{t}^{e} h\left(s_{0}, \ldots, s_{t-1}, s_{t-1} d_{t}\right)-d_{t} \Gamma_{t}^{e} h\left(s_{0}, \ldots, s_{t-1}, s_{t-1} u_{t}\right)}{u_{t}-d_{t}} \\
\bar{\beta}=\frac{\Gamma_{t}^{e} h\left(s_{0}, \ldots, s_{t-1}, s_{t-1} u_{t}\right)-\Gamma_{t}^{e} h\left(s_{0}, \ldots, s_{t-1}, s_{t-1} d_{t}\right)}{s_{t-1}\left(u_{t}-d_{t}\right)}
\end{array}\right.
$$

Thus, we obtain (2.8) for $t-1$ and the induction step follows.

We now introduce the following Cox-Ross-Rubinstein model (see [12]). The risky asset $S^{c r r}$ is defined by:

$$
\begin{aligned}
& S_{0}^{c r r}=S_{0}, \\
& S_{t+1}^{c r r}=S_{t}^{c r r} U_{t+1}^{c r r}, \quad \text { for } t \in\{0, \ldots T-1\}
\end{aligned}
$$

where the independent random variables $U_{t}^{c r r}$ are the coordinate applications defined on the probability space $\left(\Omega^{c r r}:=\prod_{t=1}^{T}\left\{u_{t}, d_{t}\right\}, \mathcal{P}\left(\Omega^{c r r}\right)\right)$. We set $\mathbb{F}^{c r r}=\left\{\mathcal{F}_{t}^{c r r} \mid t \in \mathcal{T}\right\}$ with $\mathcal{F}_{0}=\left\{\emptyset, \Omega^{c r r}\right\}, \mathcal{F}_{t}^{c r r}=\sigma\left(U_{1}^{c r r}, \ldots, U_{t}^{c r r}\right)$ for $t \in\{1, \ldots T\}$ and we define the probability $\mathbb{P}^{c r r}$ on $\mathcal{P}\left(\Omega^{c r r}\right)$ by

$$
\mathbb{P}^{c r r}\left(\left(\omega_{1}, \ldots, \omega_{T}\right)\right)=\prod_{t=1}^{T}\left(\pi_{t} \delta_{\left\{u_{t}\right\}}\left(\omega_{t}\right)+\left(1-\pi_{t}\right) \delta_{\left\{d_{t}\right\}}\left(\omega_{t}\right)\right)
$$

Proposition 2.2 can then be rewritten as follows.

Proposition 2.3. Under the assumptions of Proposition 2.2, we have for $t \in \mathcal{T}$ that

$$
\begin{aligned}
\Gamma_{t}^{e} h\left(s_{0}, \ldots, s_{t}\right) & =\mathbb{E}^{c r r}\left(h\left(s_{0}, \ldots, s_{t}, s_{t} U_{t+1}^{c r r}, \ldots, s_{t} U_{t+1}^{c r r} \ldots U_{T}^{c r r}\right)\right) \\
\Gamma_{t}^{e} h\left(S_{0}^{c r r}, \ldots, S_{t}^{c r r}\right) & =\mathbb{E}^{c r r}\left(h\left(S_{0}^{c r r}, \ldots, S_{T}^{c r r}\right) \mid \mathcal{F}_{t}^{c r r}\right) .
\end{aligned}
$$

The super-replication price of $H=h\left(S_{0}, \ldots, S_{T}\right)$ is thus the replication price of $h\left(S_{0}^{c r r}, \ldots, S_{T}^{c r r}\right)$ in the CoxRoss-Rubinstein model defined above. 
Remark 2.4. Assume that conditionally to $\mathcal{F}_{t}$, the distribution of $S_{t+1}$ is discrete and finite, as in a tree model. Then, the super-replication capital for a convex payoff is given by the CRR price of a binomial model obtained by eliminating all the "middle" branches. A similar result was found in [18] in the context of a trinomial model. A second family of models satistying Assumption 1.1 are the one such that conditionally to $\mathcal{F}_{t}$, the distribution of $\frac{S_{t+1}}{S_{t}}$ is equivalent to the Lebesgue measure on $\left[d_{t+1}, u_{t+1}\right]$. Then the super-replication capital for a convex payoff is equal to the CRR price of a binomial model whose coefficients are given by the boundaries of this support.

Note that we have explicitly obtain the (dynamic) super-replication prices and also the hedging strategy which are given by the $\bar{\beta}$.

Remark 2.5. Now we want to see what happens, when conditionally to $\mathcal{F}_{t}$, the support of the distribution of $\frac{S_{t+1}}{S_{t}}$ is $\mathbb{R}^{+}$. To do that we assume that $d_{1}=\ldots=d_{T}=0$ and $u=u_{1}=\ldots=u_{T}$ goes to $\infty$. Let us fix $t=T-1$.

$$
\Gamma_{T-1}^{e} h\left(s_{0}, \ldots, s_{T-1}\right)=h\left(s_{0}, \ldots, s_{T-1}, 0\right)+s_{T-1} \lim _{u \rightarrow \infty} \frac{h\left(s_{0}, \ldots, s_{T-1}, s_{T-1} u\right)}{s_{T-1} u}
$$

Assume that $s_{T-1} \geq 0$. For $h\left(s_{0}, \ldots, s_{T}\right)=\left(s_{T}-K\right)^{+}\left(\right.$where $\left.x^{+}=\max (x, 0)\right), \Gamma_{T-1}^{e} h\left(S_{0}, \ldots, S_{T-1}\right)=S_{T-1}$, while for $h\left(s_{0}, \ldots, s_{T}\right)=\left(K-s_{T}\right)^{+}, \Gamma_{T-1}^{e} h\left(S_{0}, \ldots, S_{T-1}\right)=K$. Thus, we get back results already present in $[10]$.

We now turn our attention to the American case.

Proposition 2.6. Let $H=\left(h_{t}\left(S_{0}, \ldots, S_{t}\right)\right)_{t \in \mathcal{T}}$ be a a bounded from below American contingent claim, where $h_{t}$ are convex functions from $\mathbb{R}^{t+1}$ to $\mathbb{R}$ and $\sup _{\tau \in \mathcal{S}_{0, T}, Q \in \mathcal{P}} E^{Q} \mid\left[H_{\tau} \mid\right]<\infty$. Let Assumption 1.1 hold true. Then

$$
\begin{aligned}
\Gamma_{T}^{a} h\left(s_{0}, \ldots, s_{T}\right) & =h_{T}\left(s_{0}, \ldots, s_{T}\right), \\
\Gamma_{t}^{a} h\left(s_{0}, \ldots, s_{t}\right) & =\max \left(\mathbb{E}^{c r r}\left(\Gamma_{t+1}^{a} h\left(s_{0}, \ldots, s_{t} U_{t+1}^{c r r}\right)\right), h_{t}\left(s_{0}, \ldots, s_{t}\right)\right) .
\end{aligned}
$$

Again the super-replication price of $H=\left(h_{t}\left(S_{0}, \ldots, S_{t}\right)\right)_{t \in \mathcal{T}}$ is the replication price of $\left(h_{t}\left(S_{0}^{c r r}, \ldots, S_{t}^{c r r}\right)\right)_{t \in \mathcal{T}}$ in the Cox-Ross-Rubinstein model defined above.

Proof. The proof is similar to the one of Proposition 2.2 as the convexity of the operator is preserved since we consider at each time step the maximum of convex functions.

\section{LINK WITH MULTIPLE-PRIORS MODEL}

We take for $S$ the canonical process $S_{t}(\omega)=\omega_{t}$ for every $\omega=\left(\omega_{0}, \ldots, \omega_{T}\right)$ in the path-space $\mathbb{R}^{T+1}, \mathcal{F}_{t}=$ $\mathcal{B}\left(\mathbb{R}^{t+1}\right), \mathcal{F}_{t}^{U}$ the universal completion of $\mathcal{F}_{t}$ and $\mathbb{F}^{U}=\left(\mathcal{F}_{t}^{U}\right)_{t \in \mathcal{T}}$. This setup allows for all possible choices of one-dimensional stock price process, since every stochastic process $S=\left(S_{t}\right)_{t \in \mathcal{T}}$ can be realized using the corresponding law on the path-space. Here we will assume that $S_{0}=s$ for a given $s \in \mathbb{R}$. In order to model the kind of uncertainty we face in the paper, we assume that for all $0 \leq t \leq T-1$ the (random) set $\mathcal{Q}_{t+1}$ which represents the law of the conditional price process at $t+1$ given the information $\omega^{t}=\left(\omega_{0}, \ldots, \omega_{t}\right) \in \mathbb{R}^{t+1}$ until time $t$ is given by

$$
\mathcal{Q}_{t+1}\left(\omega^{t}\right)=\left\{P \in \mathfrak{P}(\mathbb{R}), \operatorname{supp}(P) \subset\left[\omega_{t} d_{t+1}, \omega_{t} u_{t+1}\right]\right\},
$$

where $\mathfrak{P}(\mathbb{R})$ is the set of all probability measures on $\mathbb{R}$. The Cornerstone Assumption $(\mathrm{CA})$ in the literature on multiple-priors models (see for example [9]) asserts that for all $0 \leq t \leq T-1$

$$
\operatorname{Graph}\left(\mathcal{Q}_{t+1}\right)=\left\{\left(\omega^{t}, P\right) \in \mathbb{R}^{t+1} \times \mathfrak{P}(\mathbb{R}), P \in \mathcal{Q}_{t+1}\left(\omega^{t}\right)\right\}
$$

is an analytic set. An analytic set is the continuous image of some Polish space, see [1, Theorem 12.24 p447], see also [7, Chapter 7] for more details on analytic sets.

Proposition 3.1. For all $0 \leq t \leq T-1, \mathcal{Q}_{t+1}$ is a non-empty, convex valued random set and (CA) holds true. 
Proof. It is clear that $\mathcal{Q}_{t+1}$ is a non-empty and convex valued random set. One can easily see that

$$
\mathcal{Q}_{t+1}\left(\omega^{t}\right)=\left\{P \in \mathfrak{P}(\mathbb{R}), P=R \circ f_{t}^{-1}\left(\omega^{t}, .\right), R \in \mathcal{R}_{t+1}\right\}
$$

where $f_{t}\left(\omega^{t}, x\right)=\omega_{t} x$, for all $\left(\omega^{t}, x\right) \in \mathbb{R}^{t+1} \times \mathbb{R}$ and $\mathcal{R}_{t+1}=\left\{R \in \mathfrak{P}(\mathbb{R}), \operatorname{supp}(R) \subset\left[d_{t+1}, u_{t+1}\right]\right\}$. So, in order to check that $\operatorname{Graph}\left(\mathcal{Q}_{t+1}\right)$ is analytic, it is enough to check that $\operatorname{Graph}\left(\mathcal{R}_{t+1}\right)=\Omega^{t} \times \mathcal{R}_{t+1}$ is an analytic set (see [4, Section 2.3]). We prove that $\mathcal{R}_{t+1}$ is Borel. Let $\psi: R \rightarrow R(]-\infty, d_{t+1}[\cup] u_{t+1},+\infty[)$. Then $\psi$ is Borel-measurable (see [7, Corollary 7.26.1, p134]) and $\mathcal{R}_{t+1}=\psi^{-1}(\{0\})$. One can also use [17, Corollary $4.6]$.

The next step is to verify that the multiple-priors no-arbitrage condition (see $N A\left(\mathcal{Q}^{T}\right)$ below) holds true, where $\mathcal{Q}^{T}$ is the set of priors on $\mathbb{R}^{T+1}$ constructed from the one-period priors sets. Indeed, under (CA) one can do measurable selection and using Jankov-von Neumann Theorem (see [7](Proposition 7.49 p182)), we can define

$$
\mathcal{Q}^{T}:=\left\{q_{1} \otimes \cdots \otimes q_{T-1}, q_{s} \in \mathcal{S} K_{s}, q_{s}\left(\cdot, \omega^{s-1}\right) \in \mathcal{Q}_{s}\left(\omega^{s-1}\right) Q_{s-1} \text {-a.s. } \forall 1 \leq s \leq T-1\right\}
$$

where $\otimes$ stands for the Fubini product integration, $\mathcal{S} K_{t}$ is the set of universally-measurable stochastic kernel on $\mathbb{R}$ given $\mathbb{R}^{t}$ and $Q_{s}=q_{1} \otimes \cdots \otimes q_{s-1}$. The notation $\mathcal{Q}^{t}$ will be used when replacing in the above equation $T$ by $t$.

The $N A\left(\mathcal{Q}^{T}\right)$ states that for $\phi \in \Phi, X_{T}^{0, \phi} \geq 0 \mathcal{Q}^{T}$-q.s. implies that $X_{T}^{0, \phi}=0 \mathcal{Q}^{T}$-q.s. Here $\mathcal{Q}^{T}$-q.s. means that it holds true outside a $\mathcal{Q}^{T}$-polar set and $N \subset \Omega^{T}$ is called a $\mathcal{Q}^{T}$-polar set if for all $P \in \mathcal{Q}^{T}$, there exists $A_{P} \in \mathcal{B}_{c}(X)$ such that $P\left(A_{P}\right)=0$ and $N \subset A_{P}$.

Proposition 3.2. For all $0 \leq t \leq T-1$, assume that $d_{t+1}<1<u_{t+1}$. Then $N A\left(\mathcal{Q}^{T}\right)$ holds true.

Proof. We will prove the one-step version of the $N A\left(\mathcal{Q}^{T}\right)$ : for $\omega^{t}$ fixed in $\mathbb{R}^{t+1}$ we say that $N A\left(\mathcal{Q}_{t+1}\left(\omega^{t}\right)\right)$ condition holds true if for all $h \in \mathbb{R}$

$$
h \Delta S_{t+1}\left(\omega^{t}, \cdot\right) \geq 0 \mathcal{Q}_{t+1}\left(\omega^{t}\right) \text {-q.s. } \Rightarrow h \Delta S_{t+1}\left(\omega^{t}, \cdot\right)=0 \mathcal{Q}_{t+1}\left(\omega^{t}\right) \text {-q.s. }
$$

We proceed as in [4, Section 2.3]. Fix some $\omega^{t} \in \mathbb{R}^{t+1}$ (here (3.11) will be true on the whole space) and $h \in \mathbb{R}$. If $\omega_{t}=0$, then $\mathcal{Q}_{t+1}\left(\omega^{t}\right)=\left\{\delta_{0}\right\}$ and $h \Delta S_{t+1}\left(\omega^{t}, \cdot\right)=0 \mathcal{Q}_{t+1}\left(\omega^{t}\right)$-q.s. Assume now that $\omega_{t} \neq 0$. There exists $x^{ \pm} \in \mathbb{R}$ such that $d_{t+1} \leq x^{-}<1<x^{+} \leq u_{t+1}$. Now we choose $P^{ \pm}=\delta_{\omega_{t} x^{ \pm}}$. It is clear that $P^{ \pm} \in \mathcal{Q}_{t+1}\left(\omega^{t}\right)$ hence $P^{ \pm}\left(h \Delta S_{t+1}\left(\omega^{t}, \cdot\right) \geq 0\right)=1$ implies that $h\left(\omega_{t} x^{ \pm}-\omega_{t}\right) \geq 0$. The fact that $h=0$ follows and the $N A\left(\mathcal{Q}_{t+1}\left(\omega^{t}\right)\right)$ condition holds true for all $\omega^{t} \in \mathbb{R}^{t+1}$. Now $\left[9\right.$, Theorem 4.5] implies that the $N A\left(\mathcal{Q}^{T}\right)$ condition holds true (here $S$ is Borel measurable and use Proposition 3.1). Note that to apply this theorem it is enough to have the $N A\left(\mathcal{Q}_{t+1}\left(\omega^{t}\right)\right)$ condition on a $\mathcal{Q}^{t}$-full measure set.

From Proposition 3.1, we can apply the First Fundamental Theorem of Asset Pricing and the Superhedging Theorem, see [9, First Fundamental Theorem, Superhedging Theorem]. The First Fundamental Theorem of Asset Pricing asserts that the $N A\left(\mathcal{Q}^{T}\right)$ is equivalent to the following: for all $Q \in \mathcal{Q}^{T}$, there exists some $P \in \mathcal{M}^{T}$ such that $Q \ll P$ where

$$
\mathcal{M}^{T}:=\left\{P \in \mathfrak{P}\left(\mathbb{R}^{T+1}\right), \exists Q^{\prime} \in \mathcal{Q}^{T}, P \ll Q^{\prime} \text { and } P \text { is a martingale measure }\right\}
$$

The multiple-priors super-replication price of some european contingent claim $H$ is defined by

$$
p_{r}^{e}(H)=\inf \left\{x \in \mathbb{R}: \exists \Phi=\left(\Phi_{t}\right)_{t \in\{1, \ldots, T\}} \mathbb{F}^{U} \text {-predictable, } \mathbb{P}\left(X_{T}^{x, \Phi} \geq H\right)=1, \forall \mathbb{P} \in \mathcal{Q}^{T}\right\}
$$


If $H$ is an upper semianalytic European contingent claim (recall that a function $f: X \rightarrow \mathbb{R} \cup\{ \pm \infty\}$ is uppersemianalytic on $X$ if $\{x \in X f(x)>c\}$ is an analytic set), the Superhedging Theorem states that

$$
p_{r}^{e}(H)=\sup _{P \in \mathcal{M}^{T}} E_{P}(H)
$$

As in the mono-prior case, it is difficult to compute the super-replication price using this dual formula since the set $\mathcal{M}^{T}$ given in (3.12) is hard to characterize. A way to do it is to provide a generalisation of the CGT algorithm to the multiple-priors case, this will be adress in [11] for a general set of priors. When $\mathcal{Q}_{t+1}\left(\omega^{t}\right)$ is given by (3.10), one can easily compute the super-replication price using only the primal formulation and thus avoiding the use of $\mathcal{M}^{T}$ (and the related measurability issues). In the next proposition, we prove that the multiple-priors super-replication price reduces again to the Cox-Ross-Rubinstein price. Let $H=h\left(s, S_{1}, \ldots, S_{T}\right)$ be an European contingent claim with $h: \mathbb{R}^{T+1} \rightarrow \mathbb{R}$. First we introduce the sequence of operators which gives the minimal amount of money needed to superhedge step after step:

$$
\begin{aligned}
\bar{p}_{T}^{r} & =h, \\
\bar{p}_{t}^{r}\left(\omega^{t}\right) & =\inf \left\{x, \exists \phi \in \mathbb{R}, x+\phi \Delta S_{t+1}\left(\omega^{t}, \cdot\right) \geq \bar{p}_{t+1}^{r}\left(\omega^{t}, \cdot\right) \mathcal{Q}_{t+1}\left(\omega^{t}\right) \text {-q.s. }\right\} \omega^{t} \in \mathbb{R}^{t+1}, 0 \leq t \leq T-1
\end{aligned}
$$

We also introduce the recursive definition of $\Gamma_{t}^{r} h$ :

$$
\begin{aligned}
\Gamma_{T}^{r} h & =h \\
\Gamma_{t}^{r} h\left(\omega^{t}\right) & =\pi_{t+1} \Gamma_{t+1}^{r} h\left(\omega^{t}, \omega_{t} u_{t+1}\right)+\left(1-\pi_{t+1}\right) \Gamma_{t+1}^{r} h\left(\omega^{t}, \omega_{t} d_{t+1}\right) \omega^{t} \in \mathbb{R}^{t+1}, 0 \leq t \leq T-1,
\end{aligned}
$$

where $\pi_{t}:=\frac{1-d_{t}}{u_{t}-d_{t}}, t=1, \ldots, T$.

Proposition 3.3. Let $H=h\left(s, S_{1}, \ldots, S_{T}\right)$ be an European contingent claim, where $h: \mathbb{R}^{T+1} \rightarrow \mathbb{R}$ is a convex function. Assume that for all $0 \leq t \leq T-1, d_{t+1}<1<u_{t+1}$. Then, we get that

$$
\bar{p}_{t}^{r}=\Gamma_{t}^{r} h, \text { for all } t \in \mathcal{T} \text { and } p_{r}^{e}(H)=\bar{p}_{0}^{r}(s)=\Gamma_{0}^{r} h(s) .
$$

The multiple-priors super-replication price corresponds to $\Gamma_{0}^{e} h(s)$, which has been defined in Proposition 2.2.

Proof. Let

$$
\begin{aligned}
I= & \left\{x, \exists\left(\Phi_{t}\right)_{t \in\{1, \ldots, T\}}, \mathbb{P}\left(x+\sum_{t=1}^{T} \Phi_{t} \Delta S_{t} \geq h\left(s, S_{1}, \ldots, S_{T}\right)\right)=1, \forall \mathbb{P} \in \mathcal{Q}^{T}\right\} \\
\bar{I}= & \left\{x, \exists\left(\varphi_{t}\right)_{t \in\{1, \ldots, T\}}, x+\sum_{t=1}^{T} \varphi_{t}\left(s, s y_{1}, \ldots, s y_{1} \ldots y_{t-1}\right) s y_{1} \ldots y_{t-1}\left(y_{t}-1\right) \geq h\left(s, s y_{1}, \ldots, s y_{1} \ldots y_{T}\right),\right. \\
& \left.\forall y=\left(y_{1}, \ldots, y_{T}\right) \in \prod_{t=1}^{T}\left\{d_{t}, u_{t}\right\}\right\}
\end{aligned}
$$

where in $I$ the $\Phi_{t}$ are $\mathcal{F}_{t-1}^{U}$-predictable processes and in $\bar{I}$ the $\varphi_{t}$ are $\mathcal{F}_{t-1}$-measurable functions from $\mathbb{R}^{t} \rightarrow \mathbb{R}$. We first prove that $I \subset \bar{I}$. Let $x \in I$. Then there exist some $\mathbb{F}^{U}$-predictable process $\left(\Phi_{t}\right)_{t \in\{1, \ldots, T\}}$, such that $1=\mathbb{P}\left(x+\sum_{t=1}^{T} \Phi_{t} \Delta S_{t} \geq h\left(s, S_{1}, \ldots, S_{T}\right)\right)$, for all $P \in \mathcal{Q}^{T}$. We choose $y=\left(y_{1}, \ldots, y_{T}\right) \in \prod_{t=1}^{T}\left\{d_{t}, u_{t}\right\}$ and $z^{t-1}=\left(z_{1}, \ldots, z_{t-1}\right) \in \mathbb{R}^{t-1}$. Let $\mathbb{P}^{y}=\delta_{s} \otimes \delta_{s y_{1}} \otimes \ldots \otimes \delta_{s y_{1} \ldots s y_{T}} \in \mathcal{Q}^{T}$ and $\mathbb{P}^{z^{t-1}}=\delta_{s} \otimes \delta_{s z_{1}} \otimes \ldots \otimes \delta_{s z_{1} \ldots s z_{t-1}} \in$ $\mathfrak{P}\left(\mathbb{R}^{t}\right)$. Using $\left[7\right.$, Lemma 7.27 , p173], one can find some $\mathcal{F}_{t-1^{-}}$measurable functions $\varphi_{t}^{z}: \mathbb{R}^{t} \rightarrow \mathbb{R}$ such that 
$\Phi_{t}=\varphi_{t}^{z}\left(s, S_{1}, \ldots, S_{t-1}\right) \mathbb{P}^{z^{t-1}}$-a.s. and $1=\mathbb{P}^{y}\left(x+\sum_{t=1}^{T} \Phi_{t} \Delta S_{t} \geq h\left(s, S_{1}, \ldots, S_{T}\right)\right)$ implies that $x \in \bar{I}$.

We prove by recursion that $\bar{p}_{t}^{r}$ is convex and that for all $\omega^{t} \in \mathbb{R}^{t+1}$,

$$
\begin{aligned}
\bar{p}_{t}^{r} & =\Gamma_{t}^{r} h \\
\bar{p}_{t}^{r}\left(\omega^{t}\right) & +\Psi_{t+1}\left(\omega^{t}\right) \Delta S_{t+1}\left(\omega^{t}, \cdot\right) \geq \bar{p}_{t+1}^{r}\left(\omega^{t}, \cdot\right) \mathcal{Q}_{t+1}\left(\omega^{t}\right) \text {-q.s. } \\
\Psi_{t+1}\left(\omega^{t}\right) & =\frac{\bar{p}_{t+1}^{r}\left(\omega^{t}, \omega_{t} u_{t+1}\right)-\bar{p}_{t+1}^{r}\left(\omega^{t}, \omega_{t} d_{t+1}\right)}{\omega_{t}\left(u_{t+1}-d_{t+1}\right)} .
\end{aligned}
$$

This is true by definition at $t=T$. Assume it holds true at some $t+1$. Let $\omega^{t} \in \mathbb{R}^{t+1}$, we define

$$
\begin{aligned}
& I\left(\omega^{t}\right)=\left\{(x, \varphi), \mathbb{P}\left(x+\varphi\left(\omega^{t}\right) \Delta S_{t+1}\left(\omega^{t}, \cdot\right) \geq \bar{p}_{t+1}^{r}\left(\omega^{t}, \cdot\right)\right), \forall \mathbb{P} \in \mathcal{Q}_{t+1}\left(\omega^{t}\right)\right\} \\
& \left.\bar{I}\left(\omega^{t}\right)=\left\{(x, \varphi), x+\varphi\left(\omega^{t}\right) S_{t}\left(\omega^{t}\right)(y-1) \geq \bar{p}_{t+1}^{r}\left(\omega^{t}, S_{t}\left(\omega^{t}\right) y\right)\right), \forall y \in\left\{d_{t+1}, u_{t+1}\right\}\right\},
\end{aligned}
$$

where $\varphi$ is a $\mathcal{F}_{t}^{U}$-measurable function. Using Dirac mass as before $\left(\mathbb{P}=\mathbb{P}^{S_{t}\left(\omega^{t}\right) y}, y \in\left\{d_{t+1}, u_{t+1}\right\}\right), I\left(\omega^{t}\right) \subset$ $\bar{I}\left(\omega^{t}\right)$. Then we prove the reverse inclusion as in Lemma 2.1. Let $(x, \varphi) \in \bar{I}\left(\omega^{t}\right)$. Let $\omega^{t+1}=\left(\omega^{t}, \omega\right) \in \mathbb{R}^{t+1} \times \mathbb{R}$ be such that there exists $\lambda_{t}\left(\omega^{t+1}\right) \in[0,1]$ satisfying

$$
\frac{S_{t+1}}{S_{t}}\left(\omega^{t+1}\right)=\frac{\omega}{\omega_{t}}=\lambda_{t+1}\left(\omega^{t+1}\right) d_{t+1}+\left(1-\lambda_{t+1}\left(\omega^{t+1}\right)\right) u_{t+1} .
$$

Then by convexity of $\bar{p}_{t+1}^{r}$, we get that

$$
\begin{aligned}
\bar{p}_{t+1}^{r}\left(\omega^{t}, \omega\right) & \leq \lambda_{t+1}\left(\omega^{t+1}\right) \bar{p}_{t+1}^{r}\left(\omega^{t}, \omega_{t} d_{t+1}\right)+\left(1-\lambda_{t+1}\left(\omega^{t+1}\right)\right) \bar{p}_{t+1}^{r}\left(\omega^{t}, \omega_{t} u_{t+1}\right) \\
& \leq x+\varphi\left(\omega^{t}\right) S_{t}\left(\omega^{t}\right)\left(\lambda_{t+1}\left(\omega^{t+1}\right) d_{t+1}+\left(1-\lambda_{t+1}\left(\omega^{t+1}\right)\right) u_{t+1}-1\right)=x+\varphi\left(\omega^{t}\right)\left(S_{t+1}\left(\omega^{t+1}\right)-S_{t}\left(\omega^{t}\right)\right) .
\end{aligned}
$$

Let $\mathbb{P} \in \mathcal{Q}_{t+1}\left(\omega^{t}\right)$, as $\mathbb{P}(\operatorname{supp}(\mathbb{P}))=1$, we get that $\mathbb{P}\left(\frac{S_{t+1}}{S_{t}} \in\left[d_{t+1}, u_{t+1}\right]\right)=1$ and $(x, \phi) \in I\left(\omega^{t}\right)$.

Thus

$$
\begin{aligned}
\bar{p}_{t}^{r}\left(\omega^{t}\right) & =\inf \left\{x, \exists \varphi,(x, \varphi) \in I\left(\omega^{t}\right)\right\}=\inf \left\{x, \exists \varphi,(x, \varphi) \in \bar{I}\left(\omega^{t}\right)\right\} \\
& =\pi_{t+1} \bar{p}_{t+1}^{r}\left(\omega^{t}, \omega_{t} u_{t+1}\right)+\left(1-\pi_{t+1}\right) \bar{p}_{t+1}^{r}\left(\omega^{t}, \omega_{t} d_{t+1}\right) \\
& =\pi_{t+1} \Gamma_{t+1}^{r} h\left(\omega^{t}, \omega_{t} u_{t+1}\right)+\left(1-\pi_{t+1}\right) \Gamma_{t+1}^{r} h\left(\omega_{t}, \omega_{t} d_{t+1}\right)=\Gamma_{t}^{r} h\left(\omega^{t}\right) .
\end{aligned}
$$

Moreover $\bar{p}_{t}^{r}$ is convex as a convex combinaison of convex function. As

$$
\bar{p}_{t}^{r}\left(\omega^{t}\right)+\frac{\bar{p}_{t+1}^{r}\left(\omega^{t}, S_{t}\left(\omega^{t}\right) u_{t+1}\right)-\bar{p}_{t+1}^{r}\left(\omega^{t}, S_{t}\left(\omega^{t}\right) d_{t+1}\right)}{S_{t}\left(\omega^{t}\right)\left(u_{t+1}-d_{t+1}\right)} S_{t}\left(\omega^{t}\right)(y-1)=\bar{p}_{t+1}^{r}\left(\omega^{t}, S_{t}\left(\omega^{t}\right) y\right), \forall y \in\left\{d_{t+1}, u_{t+1}\right\}
$$

$\left(\bar{p}_{t}^{r}\left(\omega^{t}\right), \Psi_{t+1}\left(\omega^{t}\right)\right) \in \bar{I}\left(\omega^{t}\right)=I\left(\omega^{t}\right)$ and we get that (3.15) and (3.16) hold true.

When solving recursively the $2 T$ equations of $\Gamma_{t}^{r} h$, one gets that $\bar{p}_{0}^{r}(s)=\Gamma_{0}^{r} h(s)=\mathbb{E}^{c r r}\left(h\left(S_{0}^{c r r}, \ldots, S_{T}^{c r r}\right)\right)$ (see Proposition 2.3 for the definition of the Cox-Ross-Rubinstein market). When solving directly the $2^{T}$ equations appearing in inf $\bar{I}$ one also get $\mathbb{E}^{c r r}\left(h\left(S_{0}^{c r r}, \ldots, S_{T}^{c r r}\right)\right)$ (this is the same computations than in the composition of Bernoulli law in order to get a Binomial law). So

$$
\bar{p}_{0}^{r}(s)=\inf \bar{I} \leq \inf I=p_{r}^{e}(H) .
$$

Since (3.15) holds true for every $\omega^{t} \in \mathbb{R}^{t+1}$, the Fubini theorem implies for all $P=q_{1} \otimes \ldots \otimes q_{T-1} \in \mathcal{Q}^{T}$ that $\mathbb{P}\left(\bar{p}_{t}^{r}+\Psi_{t+1} \Delta S_{t+1} \geq \bar{p}_{t+1}^{r}\right)=1$ and we get that

$$
\bar{p}_{0}^{r}+\sum_{t=1}^{T} \Psi_{t} \Delta S_{t} \geq H \mathcal{Q}^{T} \text {-q.s. }
$$


Thus $\bar{p}_{0}^{r}(s) \geq p_{r}^{e}(H)$ and the proof is complete. Note that $\Psi_{t}: \mathbb{R}^{t} \rightarrow \mathbb{R}$ is continuous (because $h$ is continuous and also the $\Gamma_{t}^{r} h$ by induction) so it is $\mathcal{F}_{t-1}^{U}$-measurable and also $\mathcal{F}_{t-1}$-measurable.

\section{REFERENCES}

[1] C. D. Aliprantis And K. C. Border, Infinite Dimensional Analysis : A Hitchhiker's Guide, Grundlehren der Mathematischen Wissenschaften, Springer-Verlag, Berlin, 3rd edition, 2006.

[2] M. Avellaneda, A. Levy, And A. Paras, Pricing and hedging derivatives securities in markets with uncertain volatilities, Applied Mathematical Finance, 2 (1995), pp. 73-88.

[3] J. Baptiste, L. Carassus, and E. LéPinette, Pricing without martingale measure, Preprint, hal-01774150, (2018).

[4] D. BARTL, Exponential utility maximization under model uncertainty for unbounded endowments, ArXiv, (2016).

[5] M. Beiglböck And M. Nutz, Martingale inequalities and deterministic counterparts, Electronic Journal of Probability., 19 (95) (2014), pp. 1-15.

[6] B. Bensaid, J. P. Lesne, H. Pages, and J. Scheinkman, Derivative asset pricing with transaction costs, Mathematical Finance, 2 (1992), pp. 63-86.

[7] D. P. Bertsekas and S. Shreve, Stochastic Optimal Control: The Discrete-Time Case, Athena Scientific, 2004.

[8] P. Billingsley, Probability and Measure, John Wiley and Sons, 1986.

[9] B. Bouchard And M. Nutz, Arbitrage and duality in nondominated discrete-time models, Annals of Applied Probability, 25 (2015), pp. 823-859.

[10] L. Carassus, E. Gobet, And E. Temam, A class of financial products and models a class of financial products and models where super-replication prices are explicit, in International Symposium on Stochastic Processes and Mathematical Finance, Ritsumeikan University, Tokyo, 2006.

[11] L. Carassus, J. OblóJ, And J. Wiesel, Computing the robust superreplication price, Preprint., (2018).

[12] J. Cox, S. Ross, And M. Rubistein, Option pricing: a simplified approach., Journal of Financial Economics, 7 (1979).

[13] R. C. Dalang, A. Morton, and W. Willinger, Equivalent martingale measures and no-arbitrage in stochastic securities market models, Journal Stochastics and Stochastic Reports., 29 (1990), pp. 185-201.

[14] L. G. Epstein And S. Ji, Ambigous volatility, possibility and utility in continuous time, Journal of Mathematical Economic, 50 (2014), pp. 269-282.

[15] H. Föllmer and D. Kramkov, Optional decompositions under constraints., Probability Theory and Related Fields, 109 (1997).

[16] F. Knight, Risk, Uncertainty, and Profit, Boston, MA: Hart, Schaffner Marx; Houghton Mifflin Co, 1921.

[17] K. Lange, Borels sets of probability measures., Pacific Journal of Mathematics, 48 (1973), pp. $141-161$.

[18] C. Scagnellato and T. Vargiolu, Explicit solutions for explicit solutions for shortfall risk minimization in specific multinomial models., Decisions in Economics and Finance, 25 (2002), pp. 145-155. 